\title{
Conservación de orquídeas en una reserva privada de Palmares, Costa Rica
}

\author{
Maricela Rojas Álvarez ${ }^{1}$, Carlos O. Morales ${ }^{2}$ \& Mario A. Blanco ${ }^{2,3}$ \\ 1. Calle Ramírez, Buenos Aires de Palmares, Alajuela, Costa Rica, charithy17@gmail.com \\ 2. Escuela de Biología, Universidad de Costa Rica, 11501-2060 San José, Costa Rica, carlos.morales1264@gmail.com \\ 3. Jardín Botánico Lankester, Universidad de Costa Rica, lepanthes@hotmail.com
}

Recibido 24-VI-2016 • Corregido 18-VII-2016 • Aceptado 30-VII-2016

\begin{abstract}
Orchid conservation in a private preserve at Palmares, Costa Rica. Madre Verde Private Preserve, located in Palmares (Alajuela, Costa Rica) protects a forest relict surrounded by pastures, bushes and abandoned agriculture grounds. An inventory was conducted in this preserve to determine the orchid species diversity, prepare orchid illustration materials, analyze geographic distribution and factors affecting identified species conservation, and collect spirit and dry herbarium specimens. High quality photos from all 109 observed orchid species in 46 genera were prepared. Genera with greater species numbers are Epidendrum and Maxillaria s.l. (both with 12 spp.). Most species (86\%) are epiphytic and eight species are endemic to Costa Rica. Months with most species flowering were February, April, May, August and November during the study year 2011-2012. Geographical and altitudinal ranges from two data bases (CRBIO and IABIN) and the Costa Rica's Plant Manual were compared to the results of the present study, so we could broaden knowledge on these data for several species: 14 species were found in the preserve at higher elevations than in previous locations. Moreover, other five species were found at lower elevations than herbarium and data base records. Nine of the species observed were previously not collected in the whole Alajuela province. The most striking result was the finding of both Lepanthes guardiana and L. minutissima, which where illustrated and collected before this study only ca. 1867 , there were no ecological or precised collection data, the only illustration of the former and specimens of the latter were in Vienna Herbarium (W) and no one could heretofore find them again in Costa Rica or elsewhere. We demonstrate that even small private preserves in very disturbed areas can protect small and rare orchids and many other plant species. We recommend continuing investigation on local floras and trying to reproduce endangered species from critical landscapes, in order to avoid local or total extinction. Instruction for preserve visitors and surrounding humans populations are under the activities that can guarantee species survival in the study area.
\end{abstract}

Key words: Orchidaceae, orchids, Madre Verde Private Preserve, altitudinal range, Lepanthes guardiana, Lepanthes minutissima.
RESUMEN: La Reserva Madre Verde (RMV), ubicada en Palmares, Alajuela, Costa Rica, protege un reducto boscoso rodeado de pastizales, matorrales y terrenos de cultivo abandonados. El objetivo de este estudio fue determinar la diversidad de especies de orquídeas en la RMV mediante un inventario; además, analizamos la distribución y los factores que afectan la conservación de las especies identificadas, se prepararon especímenes (secos o en líquido) de las especies florecidas para herbario y se tomaron fotos de todas las especies; en total 109 en 46 géneros. Los géneros con mayor número de especies son Epidendrum y Maxillaria s.l. (ambos con 12 spp.). El La mayoría de especies son epífitas y ocho son endémicas en Costa Rica. Los meses con mayor floración fueron febrero, abril, mayo, agosto y noviembre. Se compararon datos de distribución geográfica y altitudinal de dos bases de datos (CRBio e IABIN) y del Manual de Plantas de Costa Rica con datos de este estudio; esto permitió ampliar el conocimiento sobre algunas especies: 14 especies se encontraron a mayores elevaciones; otras cinco especies se hallaron a menores elevaciones que las registradas en herbarios y bases de datos. Nueve especies no se habían recolectado anteriormente en toda la provincia de Alajuela. El aporte más significativo del estudio es el hallazgo de Lepanthes guardiana y L. minutissima, que fueron ilustradas y recolectadas ca. 1867, no existían hasta ahora datos ecológicos ni localidades precisas de recolecta, la única ilustración de la primera y los únicos especímenes conocidos de la segunda se hallaban en el Herbario W (Viena, Austria). Se recomienda continuar la investigación en áreas de la reserva no cubiertas por este estudio y seguir observando las especies que, según la literatura, no se esperaban en esta zona. Construir un vivero, cultivar especies de orquídeas amenazadas de extinción para la venta a visitantes y pobladores cercanos, informar a las comunidades aledañas sobre diversidad de orquídeas e importancia de la protección de flora y fauna locales, son actividades recomendadas para mantener los paisajes y las especies en la zona de estudio.

Palabras clave: Orchidaceae, orquídeas, Reserva Madre Verde, distribución altitudinal, Lepanthes guardiana, Lepanthes minutissima. 
El desarrollo de la actividad ganadera y la producción agrícola en los Montes del Agucate trajo como consecuencia la tala excesiva de los bosques, explotación de tierras poco aptas para cultivos y la pérdida de flora y fauna local. Ante este contexto, los pobladores de Palmares, en el año 2000 decidieron crear la Fundación Madre Verde (FMV) y Reserva Madre Verde (RMV), principalmente por su preocupación por el abastecimiento de agua y la calidad del aire. El área de acción de la fundación es el sector sureste del Corredor Biológico Montes del Aguacate e incluye los cantones de Atenas, San Ramón y Palmares (Alpízar, 2007; FMV, 2012).

Esta fundación trabaja en la recuperación natural de las montañas. Su plan de trabajo se sustenta en tres pilares: autosostenibilidad financiera, ambiente y cultura. Su objetivo general es recuperar y conservar tierras de vocación forestal de relevante importancia hidrológica y biológica. Forma parte del Corredor Biológico Mesoamericano en Costa Rica y sirve como puente que comunica esta área con otras iniciativas de conservación en los Montes del Aguacate. El establecimiento de la reserva es el principal logro de un proyecto llamado "Recuperación, conservación, manejo sostenible y gestión de los Montes del Aguacate" (Alpízar, 2007; FMV, 2000; Rodríguez \& Brenes, 2009).

Se han realizado numerosos proyectos para estudiar la diversidad de orquídeas de Costa Rica; entre éstos pueden citarse Pérez (1942), Rodríguez, Mora, Barahona \& Williams (1986), Atwood (1989), Atwood \& Mora $(1992,1993)$, Dressler $(1993,2003)$, Mora \& Atwood (1999), Rivera (2000), Morales (2000, 2009), Ossenbach, Ossenbach \& Pupulin (2003). Estos estudios permiten conocer cuáles especies habitan en las áreas de muestreo y mejorar la información taxonómica y ecológica sobre cada especie, con proyecciones en conservación y manejo sostenible. En la Reserva Madre Verde se han hecho varios estudios preliminares sobre temas diversos. Entre estos destaca Rodríguez (2007), con una evaluación ecológica como base para el manejo sostenible, un inventario de flora y fauna y una lista de 365 especies en 98 familias de plantas vasculares superiores. Sin embargo, esa lista incluye solamente cuatro géneros y seis especies de Orchidaceae.

El objetivo de la esta investigación fue identificar y cuantificar los géneros y las especies de orquídeas en la Reserva Madre Verde, para obtener y divulgar un registro de la diversidad en este sitio, elaborar material ilustrativo de las orquídeas, analizar distribución y factores que afectan la conservación de las especies identificadas y preparar especímenes de algunas especies florecidas para herbario.

\section{MATERIALES Y MÉTODOS}

La Reserva Madre Verde se ubica en el distrito La Granja, cantón Palmares, provincia Alajuela (1002'30" $10^{\circ} 03^{\prime} 30^{\prime \prime} \mathrm{N}, 84^{\circ} 27^{\prime} 10^{\prime \prime}-84^{\circ} 27^{\prime} 50^{\prime \prime} \mathrm{W}$ ), ca. $6 \mathrm{~km}$ al oeste del centro de Palmares (Rodríguez, 2007; Municipalidad de Palmares, 2012), en la vertiente pacífica de Costa Rica; abarca unas 40 ha y se halla en la zona de vida Bosque Muy Húmedo Premontano (Holdridge, 1978). La topografía es ondulada a escarpada, con pendientes mayores a $60 \%$; los suelos son relativamente viejos, rojos y ácidos ( $\mathrm{pH} 4,3-5,5)$. El 70\% de las tierras se recomienda para manejo forestal y zonas de protección de fauna y flora, debido a relieve, erosión y suelos poco profundos. La temperatura promedio oscila entre 18 y $21{ }^{\circ} \mathrm{C}$, con una precipitación anual promedio de 1700 a $2000 \mathrm{~mm}$ (Rodríguez, 2007).

La reserva posee potreros, matorrales y un bosque secundario (Rodríguez, 2007); de hecho, protege los últimos vestigios de los bosques naturales de la región de Palmares. Además, posee áreas y paisajes atractivos, de interés tanto turístico como educativo; incluye varios senderos de diferentes niveles de dificultad, un área para acampar, una zona recuperada por regeneración natural y reforestación, con ca. 6000 árboles sembrados, un espacio para recreación, un mariposario, un jardín de plantas medicinales, instalaciones donde operó una antigua lechería e infraestructura para recibir visitantes (FMV, 2013).

El área de estudio abarca solamente las zonas adyacentes a los senderos y al camino de entrada, más el área que rodea las instalaciones de la reserva. El muestreo se hizo al menos dos veces por mes entre enero de 2011 y julio de 2012, abarcando tres metros a cada lado de los senderos y del camino, ya que en algunas zonas el terreno es muy inclinado y es difícil buscar especies más allá de la distancia establecida. Se incluyó el espacio cercano a las instalaciones por ser un área de fácil acceso, con muchos árboles alrededor. La investigación se realizó a lo largo de ocho zonas: el camino de entrada, el sendero pequeño junto al camino de entrada que llega al mariposario, los senderos "Piedra del Zopilote" (principal), "Las Bromelias", "Sin Salida" (se une al sendero "Las Bromelias"), "Rápido" (detrás del edificio de reuniones), "La Fila" o sendero del "Mirador" (en la parte más elevada de la RMV) y el área donde se ubican las instalaciones (Fig. 1). La distancia total recorrida fue ca. $4,5 \mathrm{~km}$. El área muestreada es de unos $13500 \mathrm{~m}^{2}$. Algunos de estos recorridos se conectan entre sí. En las áreas de estudio se observaron detenidamente los árboles, el suelo y las rocas en busca de orquídeas.

Para la observación de plantas en árboles altos se utilizaron binoculares Nikon Trailblazer ATB $8 \times 42$. Se 


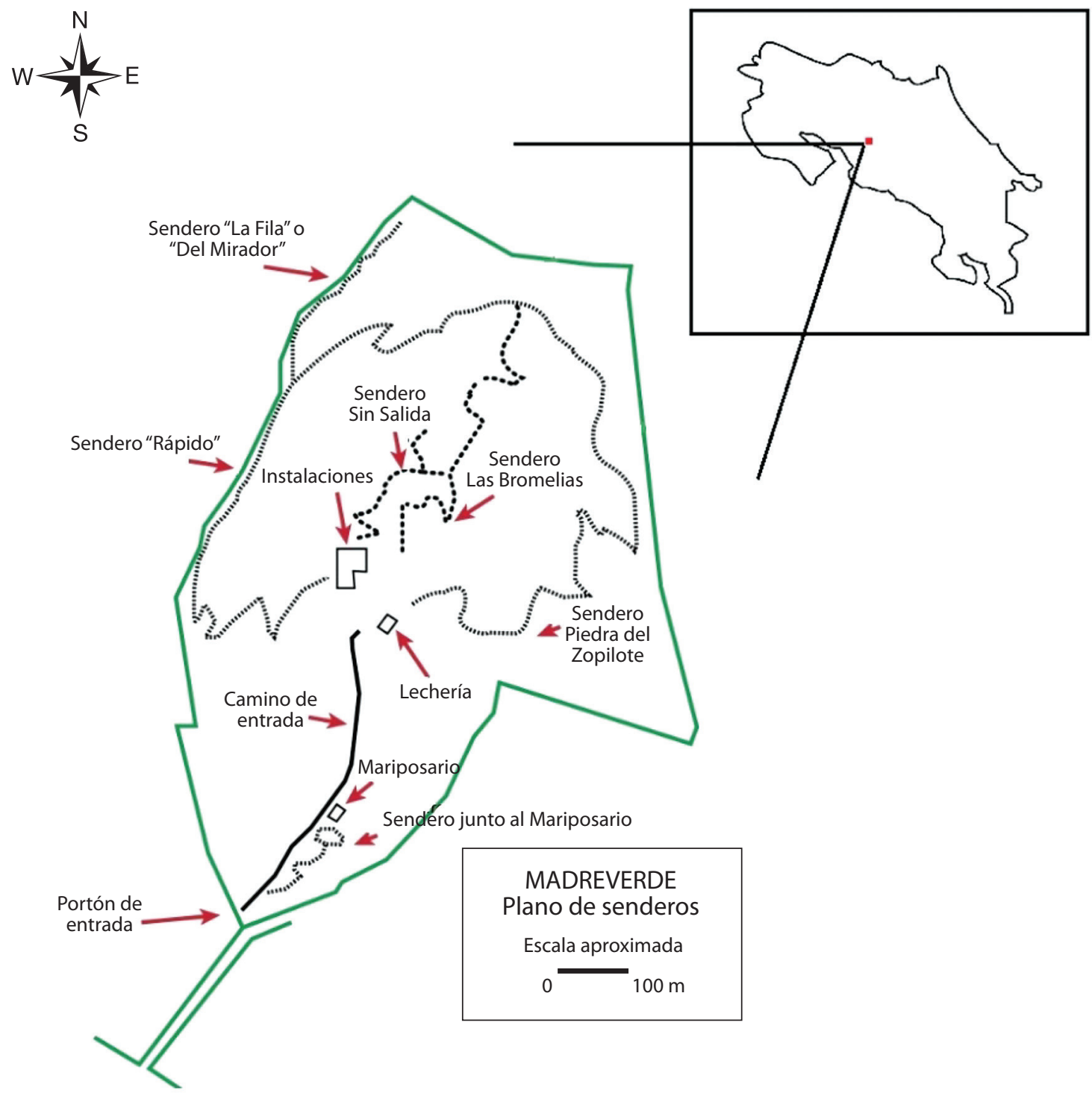

Fig. 1. Senderos de la Reserva Madre Verde (Fuente: FMV, 2013).

tomaron fotografías de las orquídeas encontradas, tanto de la planta completa como de las flores o inflorescencias, con cámaras Nikon $\$ 8000$ Coolpix y Canon 550DT2i, lentes Canon EF 18-55 mm f3.5/5.6 IS, Sigma 120-400 mm f/4-5.6 y Sigma 180 mm Macro f/3.5. Datos de elevación se tomaron con un altímetro barométrico marca Weather Master X. Las medidas de elevación con altímetros barométricos se ven afectadas por las condiciones climáticas; por tanto, para comparar datos de la RMV con los de literatura se consideró un margen de error de $\pm 50 \mathrm{~m}$. La distribución natural de las especies identificadas en este estudio se consultó en las bases de datos CRBio (2009) e IABIN (2007-2009), y en Dressler (2003). Con énfasis en la provincia de Alajuela, se anotaron nombres exactos de localidades donde se han recolectado especímenes.
Para identificar las especies se utilizó principalmente el volumen 3 del Manual de Plantas de Costa Rica (Dressler, 2003). Se decidió utilizar la nomenclatura de este manual, por considerarse el tratamiento más completo y actualizado de todas las orquídeas del país, publicado hasta ahora. Para proteger las orquídeas de la reserva, este texto no ofrece detalles de la ubicación exacta de las plantas.

\section{RESULTADOS}

Identificamos 104 especies y cinco de ellas sólo las identificamos hasta género (Cuadro 1). 
CUADRO 1

Géneros, especies y hábitat de las orquídeas

\begin{tabular}{|c|c|c|c|c|c|}
\hline Género & Especie & Háb. & Género & Especie & Háb. \\
\hline Acostaea & costaricensis Schltr. & $E$ & Brassia & arcuigera Rchb. f. & $E$ \\
\hline Campylocentrum & brenesii Schltr. & $\mathrm{E}$ & Campylocentrum & schiedei (Rchb. f.) Benth. Ex Hemsl. & $\mathrm{E}$ \\
\hline Catasetum & maculatum Kunth & $E$ & Cattleya & skinneri Bateman & $\mathrm{E}$ \\
\hline Comparettia & falcata Poepp. \& Endl. & $E$ & Cranichis & muscosa Sw. & $\mathrm{L}, \mathrm{T}$ \\
\hline Cranichis & wageneri Rchb.f. & $\mathrm{E}, \mathrm{L}, \mathrm{T}$ & Cyclopogon & elatus (Sw.) Schltr. & $\mathrm{T}$ \\
\hline Cyclopogon & sp. & $E$ & Dichaea & costaricensis Schltr. & $\mathrm{E}$ \\
\hline Dichaea & $\begin{array}{l}\text { cf. fragrantissima subsp. eburnea } \\
\text { Dressler \& Pupulin }\end{array}$ & $E$ & Dichaea & hystricina Rchb. f. & $\mathrm{E}$ \\
\hline Dichaea & poicillantha Schltr.* & $E$ & Dichaea & trulla Rchb. f. & $E$ \\
\hline Dimerandra & emarginata (G. Mey.) Hoehne & $E$ & Elleanthus & glaucophyllus Schltr. & $E$ \\
\hline Elleanthus & poiformis Schltr. & $E$ & Elleanthus & tonduzii Schltr. & $E$ \\
\hline Encyclia & ceratistes (Lindl.) Schltr. & $E$ & Encyclia & cordigera (Kunth) Dressler & $E$ \\
\hline Epidendrum & barbeyanum Kraenzl. & $\mathrm{E}$ & Epidendrum & firmum Rchb. f. & $\mathrm{E}$ \\
\hline Epidendrum & glumarum Hamer \& Garay & $\mathrm{E}$ & Epidendrum & cf. lacustre Lindl. & $E$ \\
\hline Epidendrum & cf. mora-retanae Hágsater & $E$ & Epidendrum & cf. nocturnum Jacq. & $\mathrm{E}$ \\
\hline Epidendrum & parkinsonianum Hook. & $\mathrm{E}$ & Epidendrum & radicans Pav. ex Lindl. & $\mathrm{T}$ \\
\hline Epidendrum & ramosum Jacq. & $\mathrm{E}$ & Epidendrum & sancti-ramoni Kraenzl. & $\mathrm{E}$ \\
\hline Epidendrum & sp. & $E$ & Epidendrum & stamfordianum Bateman & $E$ \\
\hline Eurystyles & standleyi Ames* & $E$ & Govenia & quadriplicata Rchb. f. & $\mathrm{T}$ \\
\hline Habenaria & eustachya Rchb. f. & $\mathrm{T}$ & Isochilus & sp. & $\mathrm{E}$ \\
\hline Jacquiniella & $\begin{array}{l}\text { aporophylla (L.O. Williams) } \\
\text { Dressler }\end{array}$ & $E$ & Jacquiniella & $\begin{array}{l}\text { teretifolia (Sw.) Britton \& P. } \\
\text { Wilson }\end{array}$ & $\mathrm{E}$ \\
\hline Laelia & rubescens Lindl. & $E$ & Leochilus & labiatus (Sw.) Kuntze & $E$ \\
\hline Leochilus & tricuspidatus (Rchb. f.) Kraenzl. & $E$ & Lepanthes & blephariglossa Schltr. & $E$ \\
\hline Lepanthes & $\begin{array}{l}\text { disticha (A. Rich. \& Galeotti) } \\
\text { Garay \& R.E. Schult. }\end{array}$ & $\mathrm{E}$ & Lepanthes & guardiana Endrés ex Luer* & $\mathrm{E}$ \\
\hline Lepanthes & inornata Schltr.* & $\mathrm{E}$ & Lepanthes & minutissima Endrés ex Luer* & $\mathrm{E}$ \\
\hline Lepanthes & sp. & $\mathrm{E}$ & Liparis & elata Lindl. & $\mathrm{T}$ \\
\hline Lockhartia & hercodonta Rchb. f. ex Kraenzl. & $\mathrm{E}$ & Masdevallia & chontalensis Rchb.f. & $\mathrm{E}$ \\
\hline Masdevallia & nidifica Rchb. f. & $E$ & Maxillaria & anceps Ames \& C. Schweinf. & $E$ \\
\hline Maxillaria & bracteata (Schltr.) Ames \& Correll & $\mathrm{E}$ & Maxillaria & bradeorum (Schltr.) L.O. Williams & $\mathrm{E}$ \\
\hline Maxillaria & dendrobioides (Schltr.) L.O. Williams & $\mathrm{E}$ & Maxillaria & fulgens (Rchb. f.) L.O. Williams & $\mathrm{E}$ \\
\hline Maxillaria & microphyton Schltr.* & $\mathrm{E}$ & Maxillaria & neglecta (Schltr.) L.O. Williams & $\mathrm{E}$ \\
\hline Maxillaria & porrecta Lindl. & $\mathrm{E}$ & Maxillaria & pseudoneglecta J.T. Atwood & $\mathrm{E}$ \\
\hline Maxillaria & scorpioidea Kraenzl. & $\mathrm{E}$ & Maxillaria & sp. & $\mathrm{E}$ \\
\hline Maxillaria & umbratilis L.O. Williams & $E$ & Mormodes & cf. horichii Fowlie & $E$ \\
\hline Nidema & boothii (Lindl.) Schltr. & $E$ & Oerstedella & centropetala (Rchb. f.) Rchb. f. & $\mathrm{E}$ \\
\hline Oerstedella & exasperata (Rchb. f.) Hágsater & $E$ & Oerstedella & pansamalae (Schltr.) Hágsater & $E$ \\
\hline Oncidium & ansiferum Rchb. f. & $\mathrm{E}$ & Oncidium & bracteatum Warsz. ex Rchb.f. & $\mathrm{E}$ \\
\hline Oncidium & obryzatoides Kraenzl.* & $\mathrm{E}$ & Pleurothallis & calyptrostele Schltr. & $E$ \\
\hline Pleurothallis & convallaria Schltr. & $E$ & Pleurothallis & cf. gelida Lindl. & $\mathrm{T}$ \\
\hline Pleurothallis & homalantha Schltr. & $\mathrm{E}$ & Pleurothallis & ramonensis Schltr.* & $\mathrm{E}$ \\
\hline Pleurothallis & ruscifolia (Jacq.) R. Br. & $\mathrm{E}$ & Pleurothallis & cf. sicaria Lindl. & $E$ \\
\hline Polystachya & foliosa (Hook.) Rchb. f. & $\mathrm{E}$ & Polystachya & masayensis Rchb. f. & $E$ \\
\hline Ponthieva & racemosa (Walter) C. Mohr & $\mathrm{E}, \mathrm{L}, \mathrm{T}$ & Prosthechea & abbreviata (Schltr.) W.E. Higgins & $E$ \\
\hline Prosthechea & fragrans (Sw.) W.E. Higgins & $\mathrm{E}$ & Prosthechea & livida (Lindl.) W.E. Higgins & $\mathrm{E}$ \\
\hline
\end{tabular}


CUADRO 1 (Continuación)

\begin{tabular}{|c|c|c|c|c|c|}
\hline Género & Especie & Háb. & Género & Especie & Háb. \\
\hline Sarcoglottis & hunteriana Schltr. & $\mathrm{T}$ & Scaphyglottis & acostae (Schltr.) C. Schweinf. & $\mathrm{E}$ \\
\hline Scaphyglottis & bidentata (Lindl.) Dressler & $E$ & Scaphyglottis & corallorhiza (Ames) Ames & $\mathrm{E}$ \\
\hline Scaphyglottis & fusiformis (Griseb.) R.E. Schult. & $\mathrm{E}$ & Scaphyglottis & imbricata (Lindl.) Dressler & $E$ \\
\hline Scaphyglottis & jimenezii Schltr. & $\mathrm{E}$ & Scaphyglottis & micrantha (Lindl.) Ames \& Correll & $\mathrm{E}$ \\
\hline Scaphyglottis & prolifera (Sw.) Cogn. & $E$ & Sigmatostalix & integrilabris Pupulin & $E$ \\
\hline Sigmatostalix & picta Rchb. f. & $E$ & Sobralia & blancoi Dressler \& Pupulin* & $E$ \\
\hline Sobralia & fragrans Lindl. & $E$ & Stanhopea & wardii G. Lodd. ex Lindl. & E. L \\
\hline Stelis & hymenantha Schltr. & $\mathrm{E}$ & Stelis & pardipes Rchb.f. & $\mathrm{E}$ \\
\hline Stenorrhynchos & lanceolatum (Aubl.) Rich. ex Spreng. & $\mathrm{T}$ & Trichocentrum & caloceras Endrés \& Rchb.f. & $\mathrm{E}$ \\
\hline Trichopilia & suavis Lindl. \& Paxton & $\mathrm{E}$ & Trichosalpinx & cedralensis (Ames) Luer & $\mathrm{E}$ \\
\hline Trigonidium & egertonianum Bateman ex Lindl. & $E$ & Trizeuxis & falcata Lindl. & $E$ \\
\hline Zootrophion & endresianum (Kraenzl.) Luer & $E$ & & & \\
\hline
\end{tabular}

* Especie endémica en Costa Rica. Háb., hábitat; E, epífita; L, litófita; T, terrestre.

Orden alfabético de izquierda a derecha y de arriba hacia abajo.

Los géneros más diversos son Epidendrum y Maxillaria s.l. (12 spp. cada uno), seguidos por Scaphyglottis (8 spp.), Pleurothallis (7 spp.), Lepanthes (6 spp.) y Dichaea (5 spp.) (Fig. 2). Los demás géneros poseen menos de cuatro especies. Los especímenes secos y las muestras preservadas en líquido (mezcla de alcohol, agua y glicerina) de 67 especies de orquídeas, recolectados en este estudio y debidamente documentados con etiquetas, se depositaron en el Herbario Luis A. Fournier (USJ) de la Universidad de Costa Rica. Todas las fotos de las orquídeas, tomadas durante los muestreos mensuales, se entregaron a los responsables de la reserva.

Como es típico en zonas tropicales, en la reserva predominan las orquídeas epífitas; 99 de las especies registradas (86\%) se encuentran en árboles o arbustos, 11 especies (10\%) son terrestres y solamente cuatro (4\%) se observan creciendo sobre rocas. No se encontró ninguna orquídea micoheterótrofa (Cuadro 1). En la reserva algunas especies de orquídeas pueden crecer en varios sustratos; es el caso de Cranichis: C. muscosa se encuentra tanto terrestre como litófita y $C$. wageneri se observa creciendo sobre rocas, suelo y árboles; Ponthieva racemosa se registra como epífita, terrestre y litófita, mientras que Stanhopea wardii crece sobre rocas y árboles (Cuadro 1).

En la Reserva se observó mayor floración en febrero, abril, mayo, agosto y noviembre (Fig. 3). Sin embargo, Dichaea trulla, Epidendrum cf. lacustre, Isochilus sp., Lepanthes sp., Liparis elata, Lockhartia hercodonta,

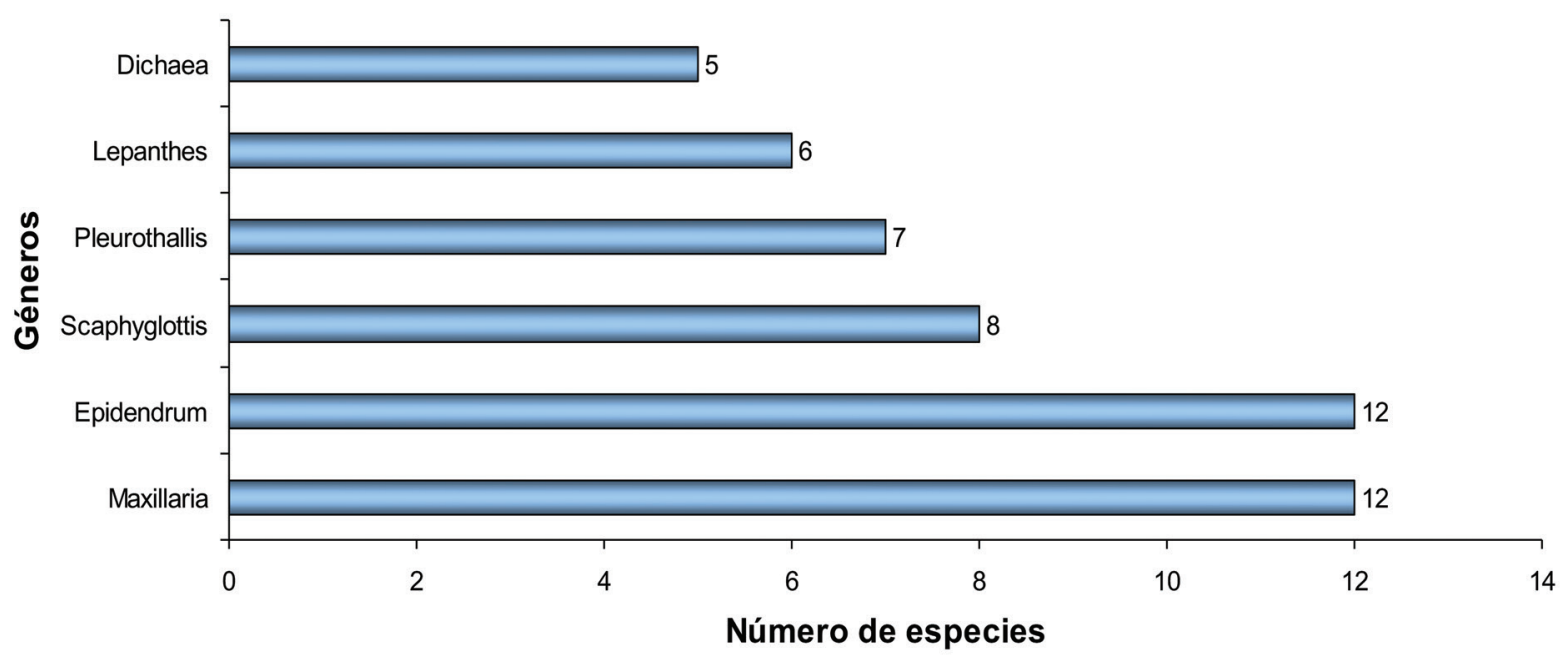

Fig. 2. Géneros con mayores números de especies en la reserva. 


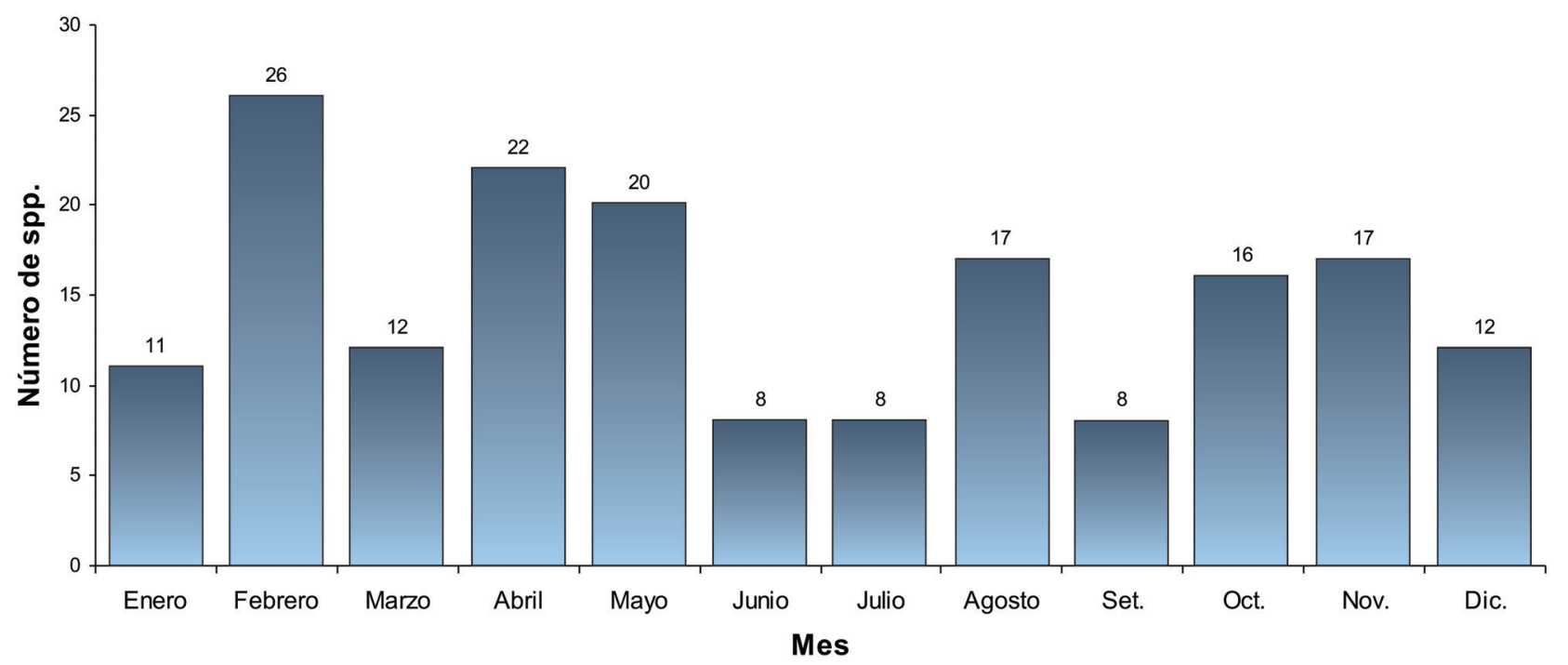

Fig. 3. Número de especies de orquídeas florecidas por mes en la Reserva Madre Verde, Costa Rica, 2011-2012.

Maxillaria fulgens, Maxillaria sp., Pleurothallis cf. gelida, $P$. cf. sicaria, Sobralia fragrans y Zootrophion endresianum no florecieron en el período de muestreo. Algunas de estas tenían restos de inflorescencias o frutos inmaduros, que permitieron identificarlas.

\section{DISCUSIÓN}

El presente estudio registra una diversidad de 109 especies de orquídeas en la Reserva Madre Verde. El único inventario previo en esta reserva (Rodríguez, 2007) solamente incluyó seis especies; por tanto, las otras 103 no se habían registrado previamente en la región de Palmares. Antes del presente estudio no se hicieron trabajos similares ni recolectas científicas de orquídeas en este cantón, posiblemente debido a que las actividades económicas en esta zona son mayormente el cultivo de café y plantas ornamentales; además, no es común que los finqueros se interesen por conocer la diversidad de flora y fauna de sus terrenos.

Los resultados obtenidos en este estudio contribuyen a mejorar el conocimiento sobre la distribución de las orquídeas registradas. Al analizar la información de Dressler (2003), CRBio (2009) e IABIN (2007-2009) sobre las especies en el país, se determinó que en estas fuentes no se registran en toda la provincia de Alajuela especímenes de nueve especies orquidáceas halladas en la RMV: Cyclopogon elatus, Epidendrum mora-retanae, Eurystyles standleyi, Govenia quadriplicata, Lepanthes blephariglossa, Oncidium ansiferum, Polystachya masayensis,
Scaphyglottis corallorhiza y Trichocentrum caloceras. Otras orquídeas, también presentes en la reserva, como Epidendrum glumarum y Pleurothallis ramonensis, se han observado en pocas zonas del país; la primera solamente cerca de la Reserva Biológica Alberto Brenes; la segunda en San Pedro de San Ramón, Reserva Biológica Alberto Brenes y Reserva Biológica Bosque de Paz.

Para especies como Catasetum maculatum, Dimerandra emarginata, Epidendrum stamfordianum, Laelia rubescens y Mormodes horichii la literatura cita hábitats y ámbitos geográficos muy diferentes a los de la RMV. Los encargados de la reserva desconocen si se trajeron de otros sitios y se cultivaron allí. Sin embargo, no puede descartarse la posibilidad de que sean especies nativas en la región de Palmares. Todas las demás especies, que no se mencionan en esta discusión (Cuadro 1), eran predecibles en la región de Palmares, porque han sido recolectadas y documentadas en sitios cercanos, o bien en otras zonas con clima y altitud similares.

Se calcula que el $12 \%$ de las especies de plantas de Costa Rica son endémicas; la mayoría de éstas se hallan en el Área de Conservación Osa y a lo largo de las cordilleras Volcánica Central, de Tilarán y de Guanacaste (INBio, 2012). Nueve de las especies registradas en este estudio son endémicas en Costa Rica: Dichaea poiciIlantha, Eurystyles standleyi, Lepanthes inornata, L. guardiana, L. minutissima, Maxillaria microphyton, Oncidium obryzatoides, Pleurothallis ramonensis y Sobralia blancoi. Esto refleja la importancia que puede tener incluso una pequeña reserva privada, como Madre Verde, en la conservación de especies vulnerables, tales como pequeñas 
orquídeas epífitas, que dependen de la existencia de bosques y parches protegidos de bosques en su ámbito geográfico natural.

La información de los especímenes de herbarios permite conocer las elevaciones máximas y mínimas en que habita una especie, pero este ámbito altitudinal puede variar cuando se exploran nuevos sitios. Esto se observó con algunas especies de este inventario: Brassia arcuigera (literatura: 500-1200 m, RMV: 1374-1442 m), Dichaea truIla (50-1200 m, RMV 1288 m), Dimerandra emarginata (01000 m, RMV 1143 m), Encyclia cordigera (0-1000 m, RMV 1198 m), Epidendrum glumarum (850-950 m, RMV 1295 m), E. stamfordianum (100-1050 m, RMV 1198 m), Laelia rubescens (0-600+ m, RMV 1202 m), Lepanthes inornata (850-1200 m, RMV 1410-1429 m), Mormodes cf. horichii (ca. 800 m, RMV $1120 \mathrm{~m}$ ), Sigmatostalix integrilabris (300$1000 \mathrm{~m}, \mathrm{RMV}$ 1239-1252 m), Sobralia fragrans (0-1050 m, RMV $1198 \mathrm{~m}$ ), Trigonidium egertonianum (0-900 m, RMV $1202 \mathrm{~m})$, Trizeuxis falcata (0-950 m, RMV 1253-1257 m) y Zootrophion endresianum (600-1200 m, RMV 1414 m) se encontraron a mayor elevación que la registrada anteriormente en Costa Rica. Además, las elevaciones a las que se encontraron Epidendrum parkinsonianum (15001600 m, RMV 1260 m), Eurystyles standleyi (1400-1500 m, RMV 1306 m), Lepanthes blephariglossa (1500-1900 m, RMV 1295 m), Scaphyglottis corallorhiza (1500-2600 m, RMV 1252 m) y Stelis hymenantha (1350-1500 m, RMV 1221-1394 m) son los registros más bajos para estas especies en este país.

En ocasiones se han documentado especies cuya información ecológica básica, como el sitio de recolecta, características del hábitat, época de floración y elevación altitudinal, no es clara o no existe. Esto sucedió con dos especies endémicas de Costa Rica, recolectadas sorpresivamente en la Reserva Madre Verde: Lepanthes guardiana (epífita, en rama gruesa con musgos, a unos $5 \mathrm{~m}$ de altura, en sitio con alta iluminación, ventilación abundante, compartiendo hábitat con Lepanthes disticha y Scaphyglottis micrantha; floración en noviembre, altitud 1316 m, Fig. 4A) y L. minutissima (epífita en árbol de jocote-Spondias purpurea, Anacardiaceae-, en ramas delgadas con algo de musgos, sitio con luminosidad intermedia a alta y abundancia de epífitas como helechos, bromelias y otras orquídeas, entre ellas Jacquiniella teretifolia, Maxillaria pseudoneglecta, M. microphyton y Acostaea costaricensis; floración en agosto, altitud 1272 m, Fig. 4B). Ambas fueron ilustradas y descritas por el recolector Augustus Endrés alrededor de 1867, pero aparte
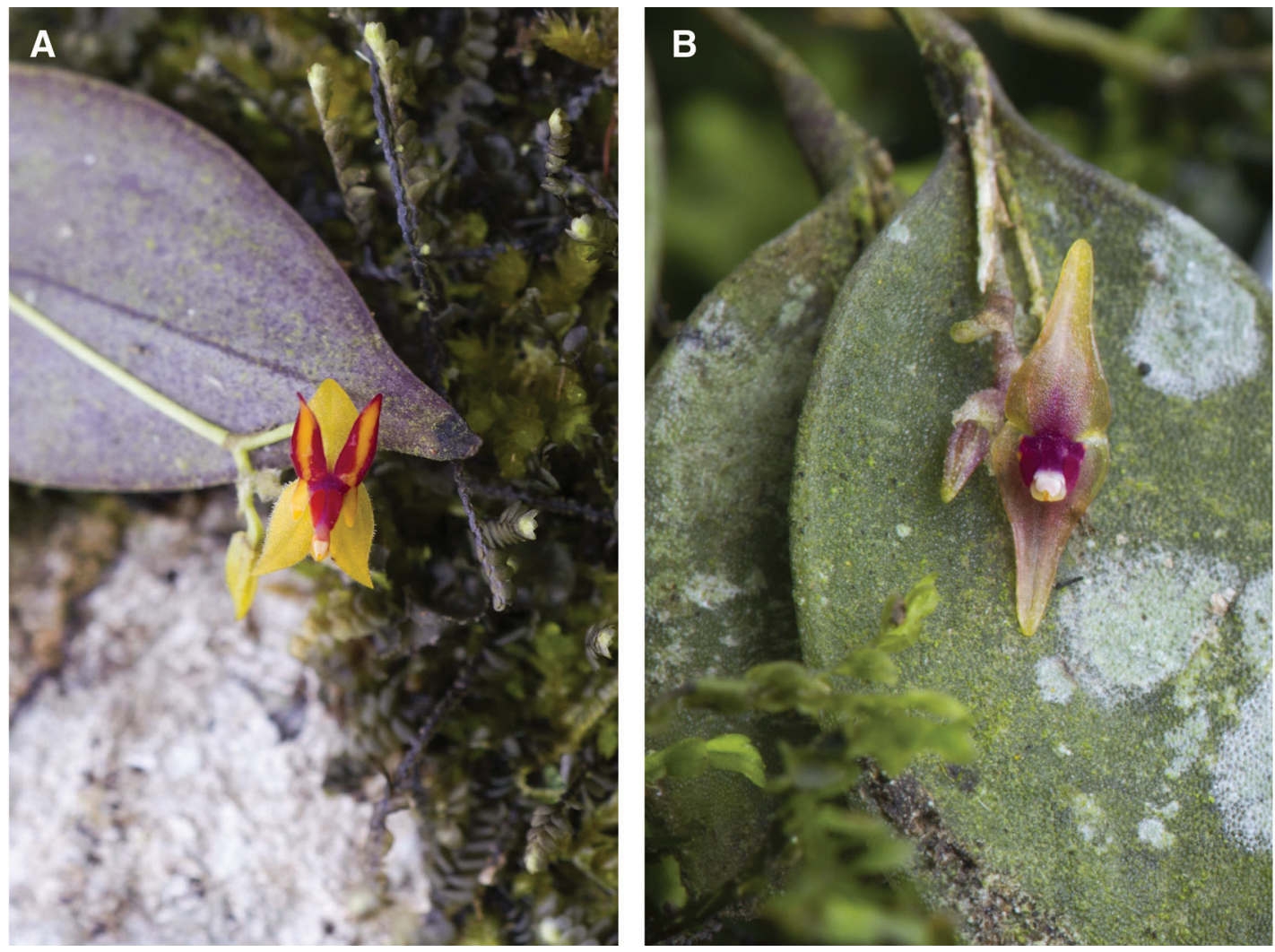

Fig. 4. Lepanthes guardiana (a); Lepanthes minutissima (b) en su hábitat natural. 
del país, él no mencionó ninguna localidad más precisa ni otra información de interés. Además, la única ilustración de la primera y los únicos especímenes conocidos de la segunda se hallaban en el Herbario W (Viena, Austria) (Luer, 1995; Dressler, 2003). Ambas especies parecían extintas, por no haber registros durante más de cien años. Aquí documentamos con fotografías originales ambas especies (Fig. 4).

Con el paso de los años, la frecuencia de observación y recolecta de algunas especies de orquídeas puede disminuir por varias razones: reducción o pérdida de hábitat por avance agrícola, ganadero y urbano, floración atractiva que motiva a algunas personas a robar las plantas para venderlas o tenerlas en casa (sin saber cultivarlas ni reproducirlas), cambio de las condiciones microclimáticas regionales, parasitismo y enfermedades, y pérdida de interés de los botánicos en estudiar áreas ecológicamente degradadas. La existencia en la RMV de las dos especies raras referidas es un aporte significativo a la orquideología de Costa Rica, porque brinda información que hasta ahora era desconocida. Según Bogarín \& Pupulin (2011), en la Cordillera de Talamanca existe una especie muy afín a L. guardiana, nombrada L. daniel-jimenezii Bogarín \& Pupulin.

Debido a extracción ilegal, algunas orquídeas se observan cada vez con menor frecuencia en numerosos ambientes naturales (Morales, 2009). Las poblaciones de Cattleya (Guarianthe) skinneri (guaria morada) se han reducido drásticamente, convirtiéndose en una especie amenazada (INBio, 2011) que actualmente sólo se puede ver en estado natural en fincas privadas de Miramar y Nicoya. Algunos jardines de Palmares y Naranjo constituyen una especie de santuario conservacionista para esta especie (García, 2012). En la RMV se encontraron varias plantas de C. skinneri y se observó propagación natural de esta especie. También se observaron con frecuencia plantas de Stanhopea wardii (torito amarillo). Según Rodríguez, Castillo \& Lizano (2012) ambas especies requieren de protección para salvar sus poblaciones, ya que tanto las guarias como los toritos son muy codiciados debido a sus flores atractivas.

Lamentablemente la RMV no está libre de extracción ilegal de especies. Durante el período de estudio desaparecieron varias orquídeas por la acción de dos posibles tipos de ladrones: en primer lugar los vendedores callejeros de plantas ("materos"), que buscan plantas fáciles de comercializar por sus flores atractivas, como Oncidium spp. (lluvia de oro), Stanhopea spp., Cattleya skinneri, Laelia rubescens (guaria de sabana) y Encyclia ceratistes; en segundo lugar los coleccionistas, con capacidad económica para invertir sumas de dinero considerables en plantas raras. Algunas de las orquídeas robadas son difíciles de identificar si no se cuenta con conocimientos sobre taxonomía vegetal; varias tienen flores poco vistosas o de tamaño pequeño. Además, cuando fueron robadas ninguna estaba en floración; entre ellas Dichaea hystricina, Maxillaria anceps y Stelis pardipes; todas crecían cerca de los senderos a poca altura del suelo. Como señala Morales (2009), si se destruyen las poblaciones originales se impide el proceso natural de reproducción, lo que puede llegar incluso a provocar la extinción local de algunas especies.

En los trabajos de mantenimiento de los senderos se cortó la vegetación alrededor de los árboles donde estaban las orquídeas referidas, con lo que quedaron más visibles y esto probablemente facilitó la acción de los ladrones. La limpieza de senderos no puede ser excesiva, porque algunas orquídeas quedan muy expuestas, mientras que otras se cortan, como ocurrió con Epidendrum cf. nocturnum, Liparis elata, Maxillaria dendrobioides, Mormodes cf. horichii y Stenorrhynchos lanceolatum. Por tanto, los encargados del mantenimiento deben ser instruidos sobre la importancia de cortar el mínimo necesario de vegetación alrededor de los senderos durante las labores de mantenimiento, para conservar un hábitat propicio para las orquídeas y todas las demás especies.

Se recomienda continuar la investigación sobre diversidad vegetal en la Reserva Madre Verde y en otras pequeñas áreas protegidas de la región, como la Reserva Ecológica La Tinajita (Rincón de Zaragoza) y la Reserva Peñas Brujas (Santiago de Palmares), para que sea una realidad el Corredor Biológico Montes del Aguacate. Se espera la colaboración de las municipalidades de los cantones involucrados: Atenas, Palmares y San Ramón.

Será interesante investigar más detalladamente las especies de orquídeas raras y otras halladas inesperadamente en la RMV. Es necesario explorar detalladamente las áreas no cubiertas por este estudio. Uno de los puntos tratados con los encargados de la reserva es la posibilidad de construir un vivero de orquídeas. Periódicamente se podrían hacer recorridos por los senderos, para recolectar plantas caídas, sembrarlas en macetas o troncos, identificarlas y rotularlas debidamente. Este vivero podría servir para educar y dar a conocer a los visitantes la diversidad de orquídeas de la zona. Además, se comentó con los encargados la posibilidad de cultivar, reproducir y vender a los visitantes plantas de Stanhopea wardii, que crece naturalmente en la reserva y es muy codiciada por sus flores grandes, atractivas y fragantes.

Otras recomendaciones para los encargados de la reserva son la publicación de datos sobre diversidad en Internet y la preparación de material informativo. 
Folletos que incluyan datos relevantes de la reserva, el trabajo conservacionista que allí se realiza e información sobre las especies de orquídeas presentes, podrían distribuirse en escuelas y colegios de Palmares y entregarse a los visitantes. También se considera importante que toda esa información se transmita a los dueños de fincas aledañas a la reserva, para incentivar la protección de flora y fauna en cerros, ríos y terrenos que ya no se dedican a actividades productivas, de modo que aumente la calidad de vida de la región tanto para especies silvestres como para poblaciones humanas.

\section{AGRADECIMIENTOS}

A los encargados de la Reserva Madre Verde, por todo el apoyo y la información que brindaron durante la investigación. A Diego Bogarín y Adam Karremans, del Jardín Botánico Lankester (Universidad de Costa Rica), por el apoyo invaluable en la identificación, especialmente de las pequeñas miniaturas epífitas. A Johnny Villarreal, Idannia Valverde y todo el personal de la Universidad Estatal a Distancia (UNED), que de múltiples maneras colaboraron para que este estudio fuera una realidad.

\section{REFERENCIAS}

Alpízar, F. (2007). Proyecto construcción de un centro de educación ambiental en el área reservada por la Fundación Madre Verde. Informe de evaluación final. Programa de Pequeñas Donaciones Costa Rica, Fondo para el Medio Ambiente Mundial. San José.

Atwood, J.T. (1989). Orchids of Costa Rica, Part 1. Icones Plantarum Tropicarum, 14, Plates 1301-1400.

Atwood, J.T. \& Mora, D.E. (1999), Orchidaceae:Tribe Maxillarieae: Subtribes Maxillariinae and Oncidiinae. In Burger, W. (Ed.), Flora Costaricensis. Fieldiana Botany, New Series, $40,1-182$.

Bogarín, D. \& Pupulin, F. (2011). Lepanthes daniel-jimenezii Bogarín \& Pupulin, spec. nov. (Pleurothallidinae: Orchidaceae), eine neue Spezies aus Costa Rica, nahe verwandt mit Lepanthes guardiana. Die Orchidee (Hamburg), 62, 111-115.

Sistema Costarricense de Información sobre Biodiversidad (CRBio). (2009). URL: http://crbio.cr/portalCRBio/ occurrences/

Dressler, R. (1993). Field Guide to the Orchids of Costa Rica and Panama. Ithaca, New York: Cornell University Press.

Dressler, R. (2003). Orchidaceae. En Hammel, B.E., Grayum, M.H., Herrera, C. \& Zamora, N. (eds.), Manual de Plantas de Costa Rica. Vol. 3. Monocotiledóneas
(Orchidaceae-Zingiberaceae). Monographs in Systematic Botany from the Missouri Botanical Garden, 93, 1-595.

Fundación Madre Verde (FMV). (2000). Proyecto de recuperación y conservación de microcuencas y terrenos de vocación forestal en Palmares y cantones vecinos. Palmares: Fundación Madre Verde.

Fundación Madre Verde (FMV). (2012). La Reserva, Inicios de la Fundación Madre Verde. URL: http://www.madreverde. com/fundacion/la-reserva

Fundación Madre Verde (FMV). (2013). Plan estratégico 20132018. URL: http://www.madreverde.com/images/stories/Plan\%20Estrategico\%202013-2018.pdf

García, J. (2012). Breve historia sobre la guaria morada. Asociación Costarricense de Orquideología (ACO). URL: http://www.ticorquideas.com/guaria_morada.htm

Holdridge, L. (1978). Ecología basada en zonas de vida. San José: Instituto Interamericano de Cooperación para la Agricultura (IICA).

IABIN. (2007-2009). Red Interamericana de Información sobre Biodiversidad. URL: http://ara.inbio.ac.cr/SSTN-IABIN/ occurrences/

Instituto Nacional de Biodiversidad (INBio). (2011). Guarianthe skinneri (Bateman) Dressler \& W.H. Higgins. URL: http://darnis.inbio.ac.cr/ubis/FMPro?-DB=UBIPUB. fp3\&-lay=WebAll\&-error=norec.html\&-Format=detail. html\&-Op=eq\&id=4004\&-Find

Instituto Nacional de Biodiversidad (INBio). (2012). Especies endémicas. URL: http://www.inbio.ac.cr/estrategia/ Estudio_2004/Paginas/diversidad02.html

Luer, C.A. (1995). New species of Lepanthes (Orchidaceae) from Costa Rica. Lindleyana, 10, 133-173.

Mora, D.E. \& Atwood, J.T. (1992). Orchids of Costa Rica, Part 2. Icones Plantarum Tropicarum, 15, Plates 1401-1500.

Mora, D.E. \& Atwood, J.T. (1993). Orchids of Costa Rica, Part 3. Icones Plantarum Tropicarum, 16, Plates 1501-1600.

Morales, F. (2000). Orquídeas, cactus y bromelias del bosque seco. Heredia: Edit. INBio.

Morales, F. (2009). Orquídeas de Costa Rica. Vol. 1-5. Heredia: Edit. INBio.

Municipalidad de Palmares. (2012). Turismo en nuestro cantón. URL: http://www.palmares.go.cr/index. php?option=com_content\&view=article\&id=2337\&lte mid $=2245$

Ossenbach, C., Ossenbach, M. \& Pupulin, F. (2003). Catálogo preliminar de las Orchidaceae de la Zona Protectora Cerros de la Carpintera, Costa Rica. Lankesteriana, 7, 127-132.

Pérez, A. (1942). Las orquídeas de Costa Rica. San José: Imprenta Española Soley y Valverde.

Rivera, G. (2000). Orquídeas: generalidades y cultivo. San José: Edit. UNED. 
Rodríguez, R.L., Mora, D.E., Barahona, M. \& Williams, N. (1986). Géneros de orquídeas de Costa Rica. San José: Edit. UCR.

Rodríguez, C. (2007). Evaluación ecológica de la Reserva Madre Verde (Palmares 2005-2006) como base para su manejo sostenible. (Tesis inédita de Maestría en Desarrollo Sostenible). Universidad de Costa Rica, San Ramón, Alajuela.
Rodríguez, C. \& Brenes, L. (2009). Fundación Madre Verde: trabajando en Palmares. Ambientico, 191, 12-14.

Rodríguez, Z., Castillo, A. \& Lizano, E. (2012). La subtribu Stanhopeinae. Asociación Costarricense de Orquideología. URL: http://www.ticorquideas.com/articulo2.htm 